\title{
EchoGéo
}

22 | 2012

Varia

\section{Moscou, la capitale de la Russie à l'ère de la compétition métropolitaine}

\section{Élisabeth Bonnet-Pineau et Aurélien Delpirou}

\section{OpenEdition}

\section{Journals}

\section{Édition électronique}

URL : https://journals.openedition.org/echogeo/13293

DOI : 10.4000/echogeo.13293

ISSN : 1963-1197

\section{Éditeur}

Pôle de recherche pour l'organisation et la diffusion de l'information géographique (CNRS UMR 8586)

Référence électronique

Élisabeth Bonnet-Pineau et Aurélien Delpirou, « Moscou, la capitale de la Russie à l'ère de la compétition métropolitaine », EchoGéo [En ligne], 22 | 2012, mis en ligne le 30 janvier 2013, consulté le 31 juillet 2021. URL : http://journals.openedition.org/echogeo/13293 ; DOI : https://doi.org/10.4000/ echogeo.13293

\section{Ce document a été généré automatiquement le 31 juillet 2021.}

EchoGéo est mis à disposition selon les termes de la licence Creative Commons Attribution - Pas d'Utilisation Commerciale - Pas de Modification 4.0 International (CC BY-NC-ND) 


\title{
Moscou, la capitale de la Russie à l'ère de la compétition métropolitaine
}

\author{
Élisabeth Bonnet-Pineau et Aurélien Delpirou
}

Si au cours de l'histoire, Moscou a subi des métamorphoses brutales, depuis 1991, la mutation accélérée de la capitale répond-elle à l'ambition de devenir un pôle urbain international tant économique que politique ? Confrontée à l'économie de marché, la ville est devenue un réceptacle d'une mutation économique anarchique en même temps qu'un laboratoire du changement. La mondialisation constitue un puissant facteur de refondation urbaine, tout particulièrement dans les quartiers centraux de Moscou. Cette ambition n'empêche pas, bien au contraire, de réintroduire des signes de « russité » tant le désir des autorités est grand pour tenter d'enraciner leur légitimité dans le passé. Phénomène récurrent en Russie à chaque changement de régime. Ainsi tandis que Moscou s'internationalise, elle se doit de marquer l'espace urbain de signes typiquement russes. 


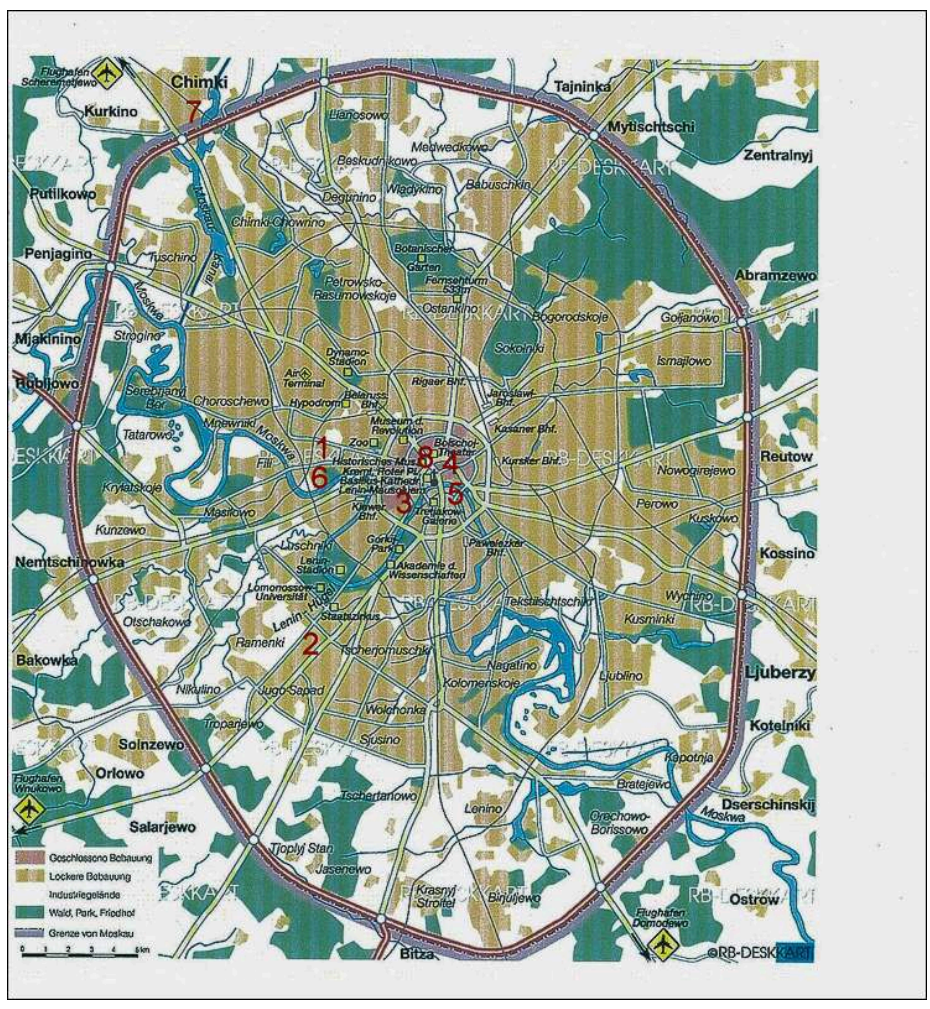

2 Les clichés présentés dans ce dossier ${ }^{1}$ (et localisés sur le plan ci-dessus) permettent d'aborder 3 thèmes :

- La métamorphose de Moscou: une imitation des grandes villes occidentales (illustrations 1 et 2)

- Entre héritage et besoin d'espace constructible (illustrations 3 à 5)

- Une métropolisation à la russe (illustrations 6 à 8 ).

\section{Illustration 1 - Réhabilitation et modernisation : la rue Arbat}

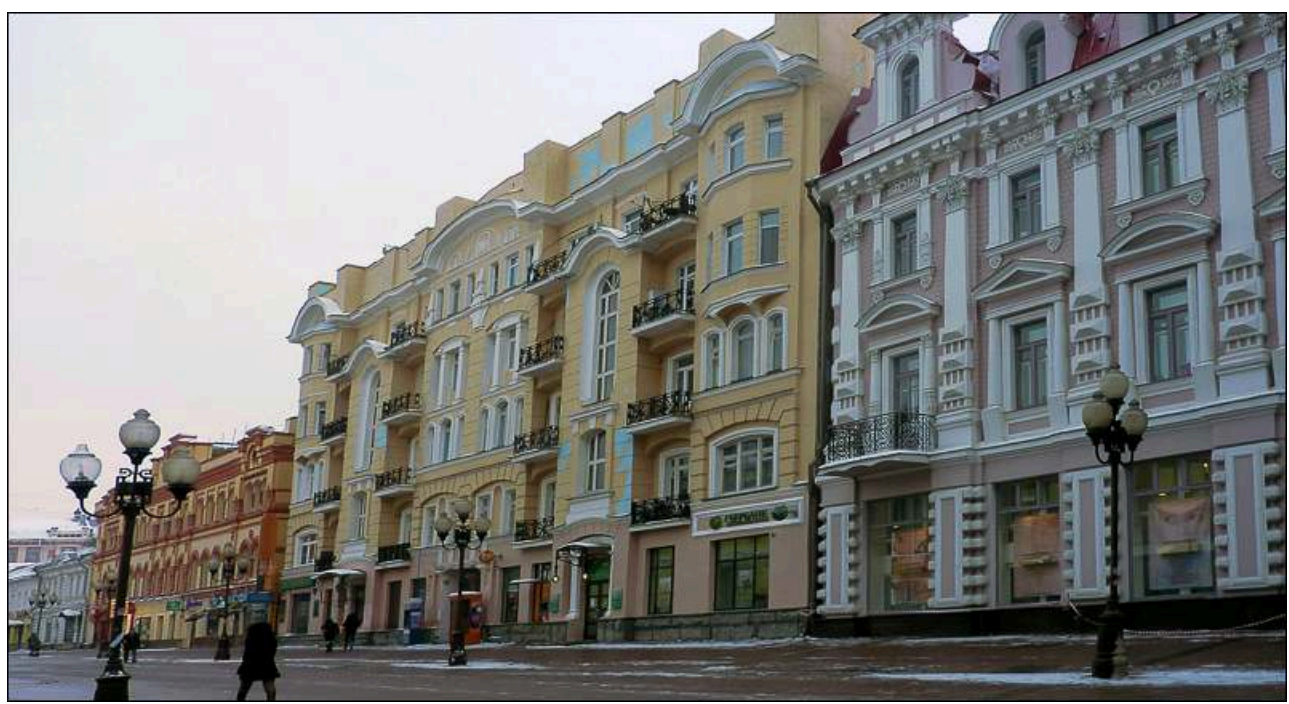

Auteur : E. Bonnet-Pineau, février 2012

3 A l'extérieur de la ville protégée par des remparts successifs et structuré par des artères radiales telles que celle de l'Arbat en direction de Smolensk, le quartier de 
l'Arbat fut l'un des lieux d'élection de la noblesse qui y séjournait l'hiver en signe de résistance à Pierre le Grand.

4 Après le grand incendie de 1812, Moscou fut reconstruite selon un plan arrêté en 1817. Le fait que la terre fut propriété d'État facilita le projet. Un nouveau type d'habitation, hôtel particulier le plus souvent à deux étages (l'osobniak) de style empire devint à la mode. La société moscovite s'y réunissait en cercles ou y tenait salon. De nombreux représentants de l'intelligentsia y vécurent au XIXe siècle, notamment Pouchkine et Dostoïevski. Aujourd'hui, les façades restaurées de ces anciens hôtels particuliers montrent leurs colonnes, leurs pilastres et leurs frontons rehaussés par les couleurs pastel, rose et gris sur la photo mais aussi jaune, verte ou bleu-vert. Profusion de couleurs comprise comme un manifeste contre la grisaille de la ville soviétique.

5 La rue Arbat est à présent décorée de grands lampadaires qui furent installés en 1986. Rue piétonne pittoresque, le vieil Arbat est l'une des rues les plus touristiques de Moscou. Les Moscovites, surtout les jeunes, sortent dans la rue pour se promener ou s'asseoir dans un café.

6 Il faut distinguer le Vieil Arbat du Nouvel Arbat construit dans le prolongement du premier sous le nom d'Avenue Kalinine bordée de gratte-ciel de béton, de verre et d'acier, symbole de la période khrouchtchévo-brejnevienne, illustration d'une des modernisations les plus brutales justifiée par la nécessité de rattraper et même de dépasser les pays occidentaux.

Illustration 2 - Privatisation et fragmentation : un immeuble en brique des années 1960

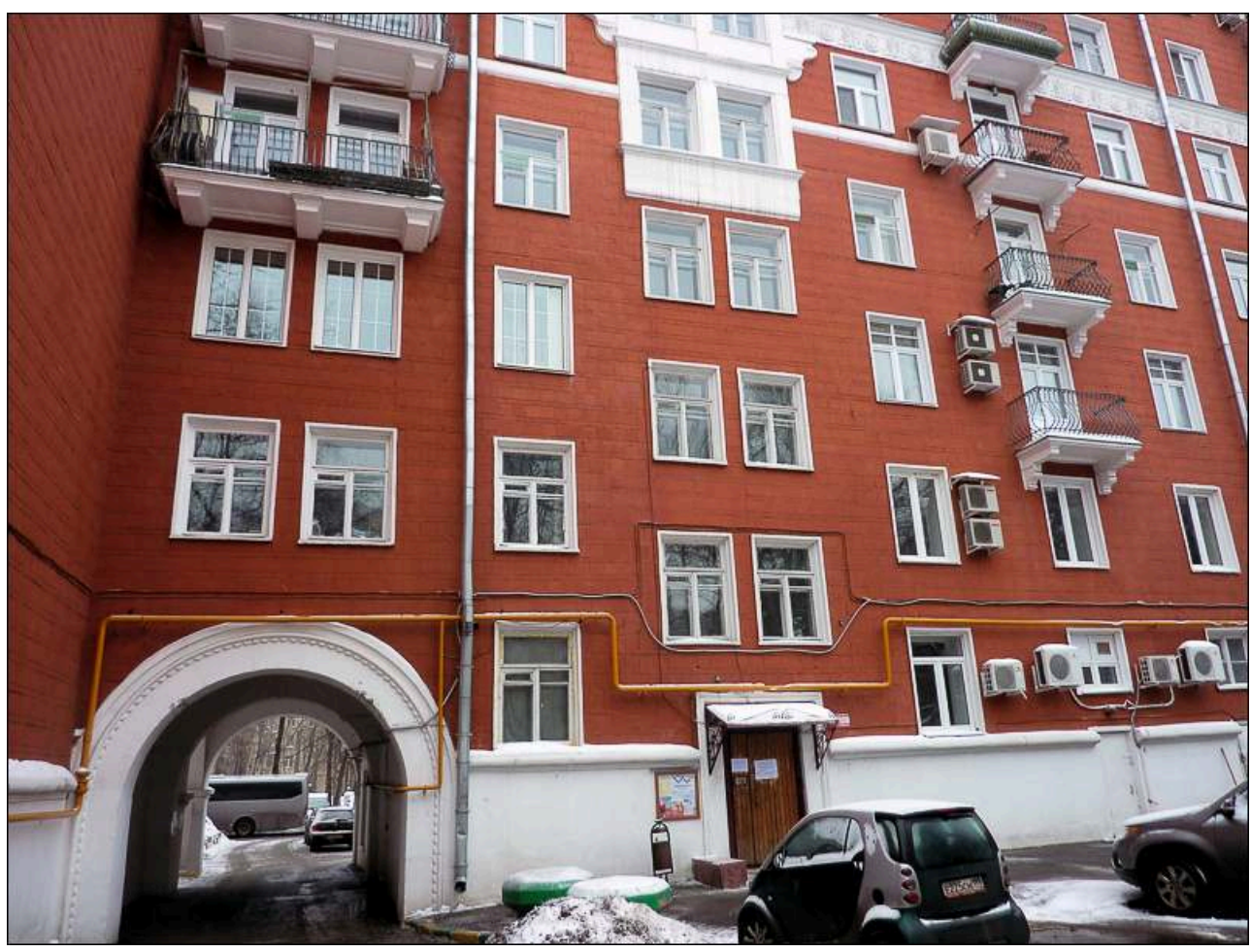

Auteur : E. Bonnet-Pineau, février 2012

7 Au sud-ouest de l'anneau des Boulevards mais à l'intérieur de la MKAD (le périphérique autoroutier de Moscou), plusieurs ensembles d'immeubles en brique construits dans les années 1960 ont été proposés à l'accession à la propriété lors du passage à l'économie 
privée au cours des années 1990. La privatisation du parc de logements a fait émerger immédiatement un marché immobilier. La population résidente enregistrée a pu ainsi devenir propriétaire à des conditions très favorables.

Procédant de la conception soviétique du micro-raïon (unité d'immeubles collectifs de 10000 habitants environ), nouvel avatar de la cité-jardin, les bâtiments, en fait plusieurs immeubles en brique, sont séparés par des aires souvent arborées, équipées de jeux pour les enfants, et par des rues intérieures le long desquelles les habitants garent leur véhicule.

9 L'immeuble de bonne facture visible sur la photo offre l'exemple d'une mise aux normes de confort et d'équipements le plus souvent défaillants: double vitrage de rigueur, conduites de chauffage au gaz courant en façade extérieure, climatiseurs pour lutter contre la chaleur des étés. La porte d'entrée n'est pas d'origine ; c'est une porte blindée avec interphone témoignant des années d'insécurité de la décennie 90. La photo ne montre pas les magasins de commerce de proximité qui sont maintenant assez fréquents en rez-de-chaussée et donnant sur les boulevards extérieurs.

Un tel quartier est devenu aujourd'hui prisé. En effet, il offre des logements assez spacieux qui ouvrent sur des cours, synonyme de tranquillité puisqu'en retrait des bruits de la ville. Ilest, de plus, bien relié au centre de Moscou (le taux de motorisation des ménages des classes moyennes qui a explosé ces dernières années facilite l'accès au centre).

11 L'accélération des mutations a renforcé les écarts de prix de l'immobilier entre quartiers. Ce groupe d'immeubles se situe dans une dynamique de renchérissement des prix au $\mathrm{m} 2$ supérieur à la moyenne sans atteindre ceux de l'ouest et du nord-ouest beaucoup plus prisés.

Illustration 3 - L'hôtel Radisson Royal, l'une des « sept sœurs » : un héritage stalinien marqueur du paysage moscovite

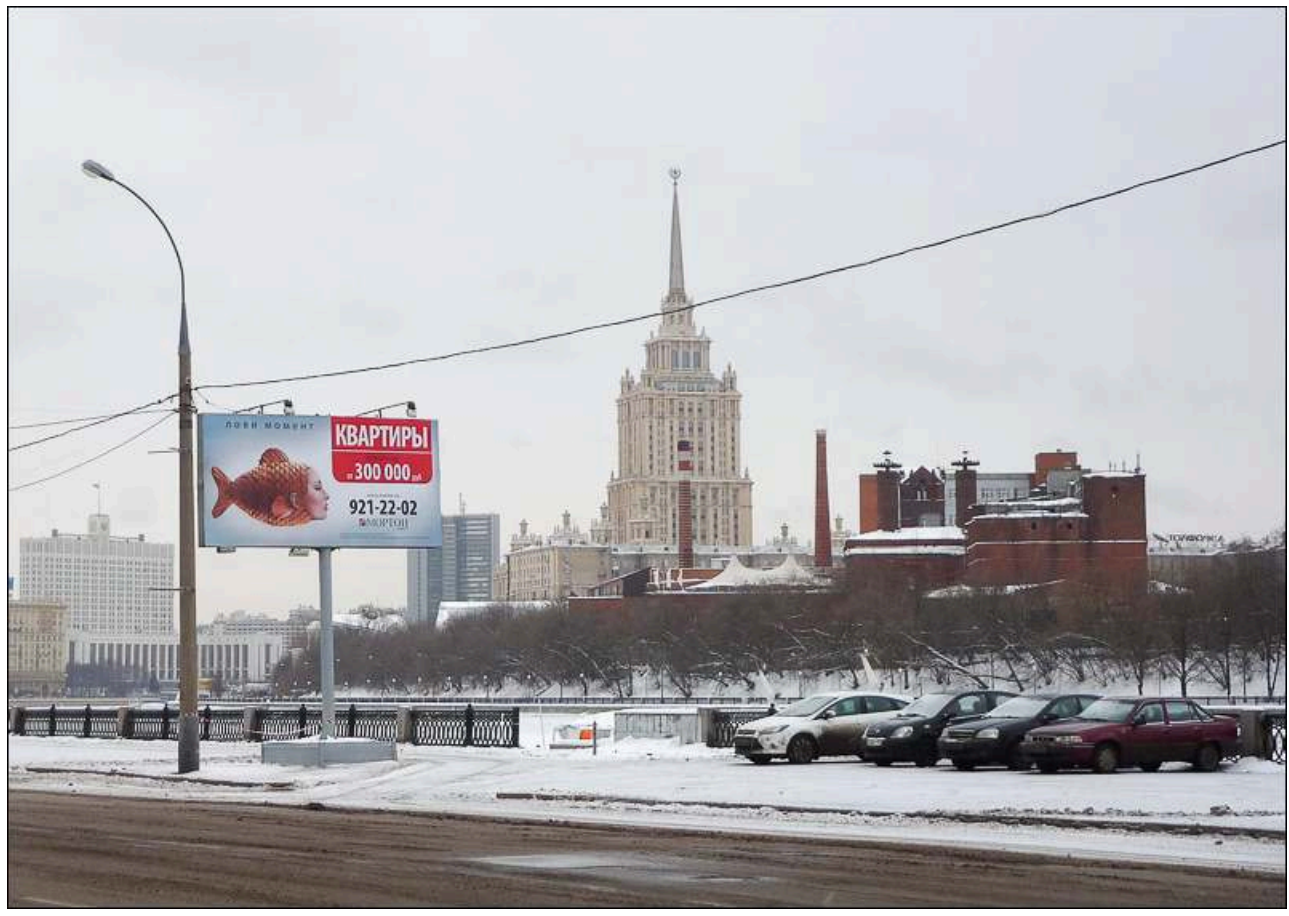

Auteur : E. Bonnet-Pineau, février 2012 

nombreux édifices religieux parmi lesquels l'église du Christ-Sauveur (en 1931) et la cathédrale Notre-Dame-de-Kazan (en 1936) si bien que la silhouette de la ville (skyline) fut profondément modifiée. La destruction des clochers fit disparaître l'un des caractères les plus emblématiques de Moscou. Après la Grande Guerre Patriotique (1941-1945), un nouvel urbanisme introduisit la «symbolique de l'empire moscovite » (Nivat G., 2007, p. 89).

Ainsi, pour célébrer les 800 ans de la ville en 1956, sept immeubles ont été construits dans un style reprenant les traditions architecturales des palais du Kremlin. L'apparition de grands immeubles dont l'architecture se voulait continuatrice de l'urbanisme de l'ancienne Russie devait compenser les pertes et destructions. L'édification des sept célèbres gratte-ciel «staliniens ", familièrement surnommés les « sept sœurs, parmi lesquelles on trouve le ministère des Affaires étrangères, celui du commerce extérieur et l'université de Moscou. La photographie montre l'ancien hôtel Ukraine devenu Radisson Royal dont la silhouette se profile au second plan, un peu à droite de l'illustration.

\section{Illustration 4 - La friche de l'ancien Hôtel Rossïa}

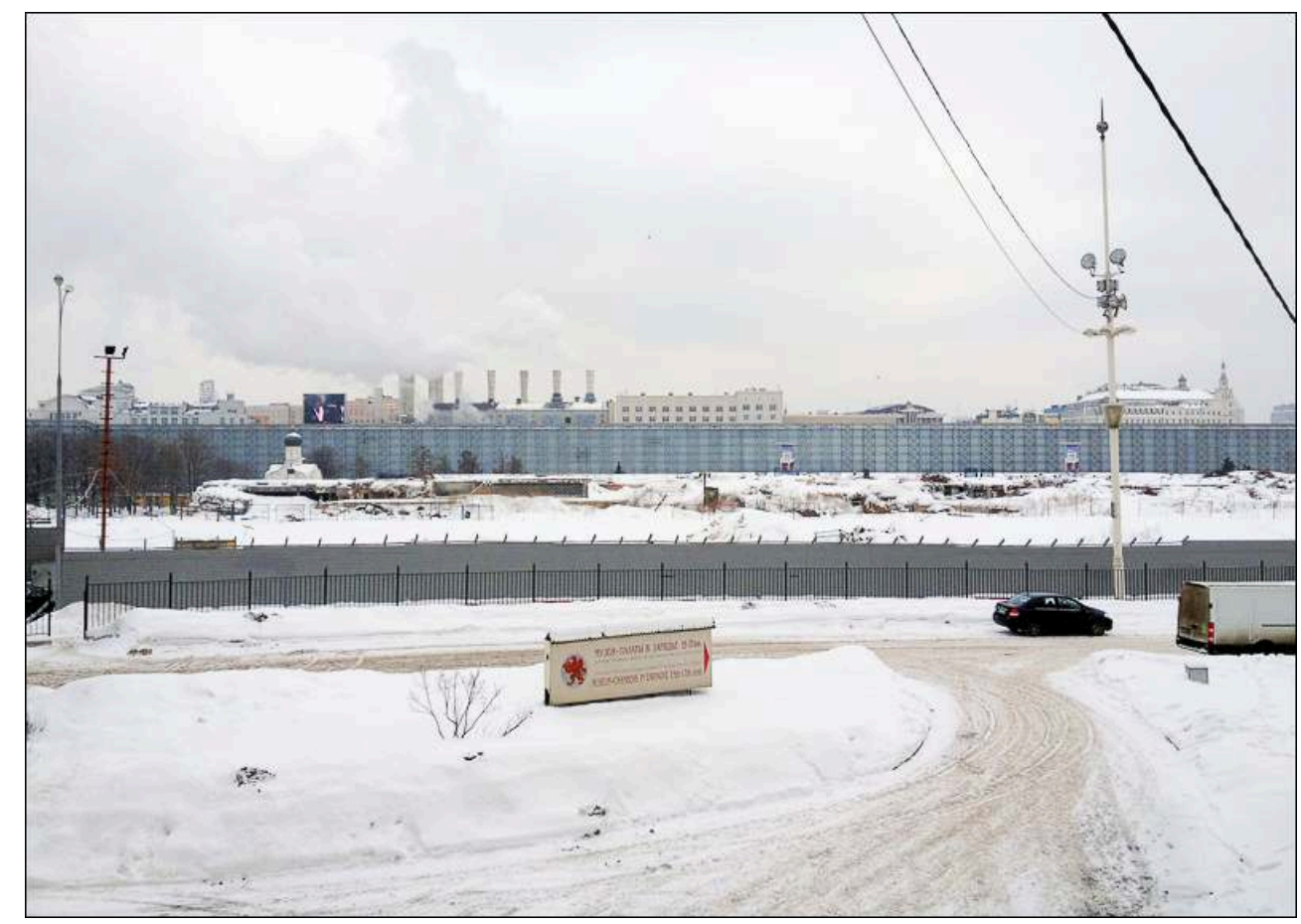

Auteur : A. Delpirou, février 2012.

Entre la prestigieuse rue Varvarka et la Moskova, à proximité immédiate de la place Rouge, cet immense terrain vague occupe l'espace libéré par la démolition de l'hôtel Rossïa. Construit en 1967 à la suite de l'éradication d'un quartier populaire jugé insalubre (Zariadie), ce bâtiment imposant et austère incarnait, avec d'autres, la puissance du régime communiste. Il abritait, en effet, près de 3200 chambres, qui accueillaient des personnalités soviétiques et internationales, mais aussi un bureau de poste, une salle de concert et un cinéma. Après la désintégration de l'URSS, ce bloc de 
béton est apparu trop symbolique pour se faire oublier et a été à son tour démoli en 2007, quarante ans après son inauguration.

Moscou est encore ponctuée de vastes alvéoles laissées par la disparition de monuments emblématiques du régime. Mais il ne faut pas se fier à l'aspect chaotique et délabré du site : par son ampleur (13 hectares) et son emplacement stratégique, ce terrain est aujourd'hui l'un des plus chers du monde et suscite bien des convoitises.

En janvier 2012, le Premier ministre Vladimir Poutine - réélu président deux mois plus tard - annonce à la surprise générale la décision d'aménager le site en grand parc urbain multifonctionnel. Non seulement cette option écarte les projets immobiliers les plus ambitieux, mais elle s'accompagne de l'ouverture d'un concours international. Les 116 propositions retenues par un jury d'experts sont présentées aux Moscovites pendant l'été 2012 ; les habitants ont même la possibilité de voter pour leur projet favori sur le site internet du Comité de Moscou de l'architecture. Dans une ville particulièrement polluée et en manque d'espaces verts, la démarche a été accueillie avec un certain enthousiasme. Mais nul ne sait encore vraiment quelle place sera accordée à ce geste inédit de démocratie participative.

Illustration 5 - Le complexe culturel Krasny Oktiabr (Octobre rouge) : une expérience pionnière de reconversion industrielle

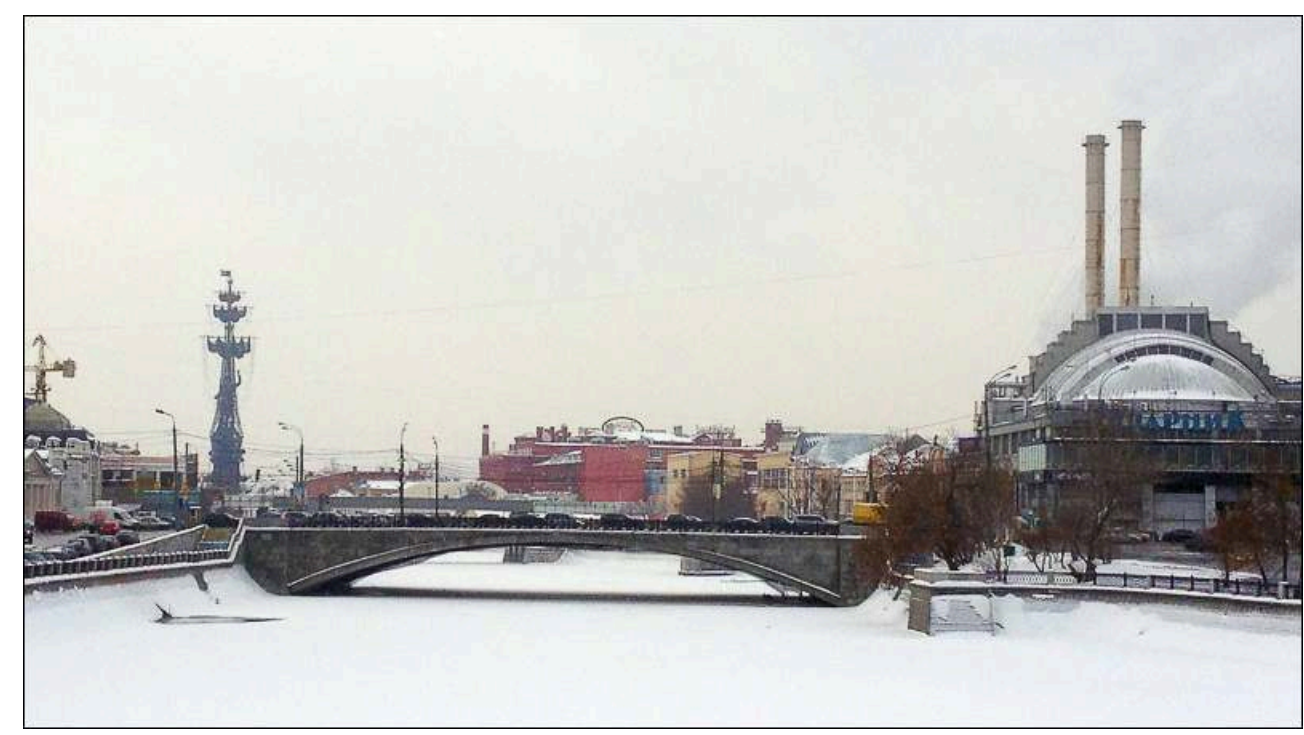

Auteur : A. Delpirou, février 2012.

Ce cliché montre, au deuxième plan, le site de l'ancienne usine Krasny Oktiabr (Octobre Rouge). Fondée en 1867, cette confiserie fut le fournisseur officiel de la Cour impériale avant de devenir l'une des fabriques les plus renommées d'URSS. Située sur une île de la Moskova, non loin du Kremlin, l'usine a connu depuis 2007 une reconversion en «cité de la création ». Elle accueille désormais une école d'architecture, des rédactions de magazines et de chaînes de télévision locales, des galeries de photos et d'art contemporain, des designers, des publicitaires. La nuit, clubs et restaurants en font l'un des lieux les plus prisés de la jeunesse moscovite.

Octobre Rouge est à la fois un cas banal et singulier. D'un côté, cette transformation s'inscrit dans des dynamiques propres à toutes les grandes métropoles internationales : développement des industries de création, valorisation du patrimoine industriel, 
affirmation de la «classe créative » dans la société urbaine. D'un autre côté, elle fait figure d'exception dans une ville où la désindustrialisation s'était jusqu'alors accompagnée d'une politique de la "table rase» au service de la spéculation immobilière : les vieilles usines du centre de Moscou ont été massivement détruites et remplacées par des bureaux et des appartements de prestige. Octobre Rouge était promis à ce destin. Mais la crise financière de 2008 a contraint les promoteurs, confrontés à la contraction de leurs moyens et à l'effondrement de la demande immobilière, à abandonner leurs projets au profit d'une réhabilitation assortie d'une mise en location. La relative modestie des loyers, dans l'une des villes les plus chères du monde, et le cachet architectural des bâtiments ont fait le reste.

Transformation pionnière ou expérience sans lendemain? Des projets similaires ont vu le jour dans d'autres quartiers, comme l'Arbat. Mais, pour l'heure, le potentiel moscovite en matière de reconversion industrielle est encore colossal : lorsque l'URSS s'est effondrée, $25 \%$ du territoire de la ville était classé « zone industrielle ». Des projets similaires ont vu le jour dans d'autres quartiers, comme l'Arbat. Mais contrairement à ce qui peut être observé en Europe occidentale, aux États-Unis ou même en Chine, où les pouvoirs publics ont fait du développement des clusters artistiques un élément de la compétition métropolitaine, les sites moscovites ne bénéficient d'aucun soutien politique et évoluent sans véritable stratégie. Au risque de devenir de véritables ghettos d'artistes, déconnectés du reste de la cité.

Illustration 6 - Moskva City : le Manhattan russe

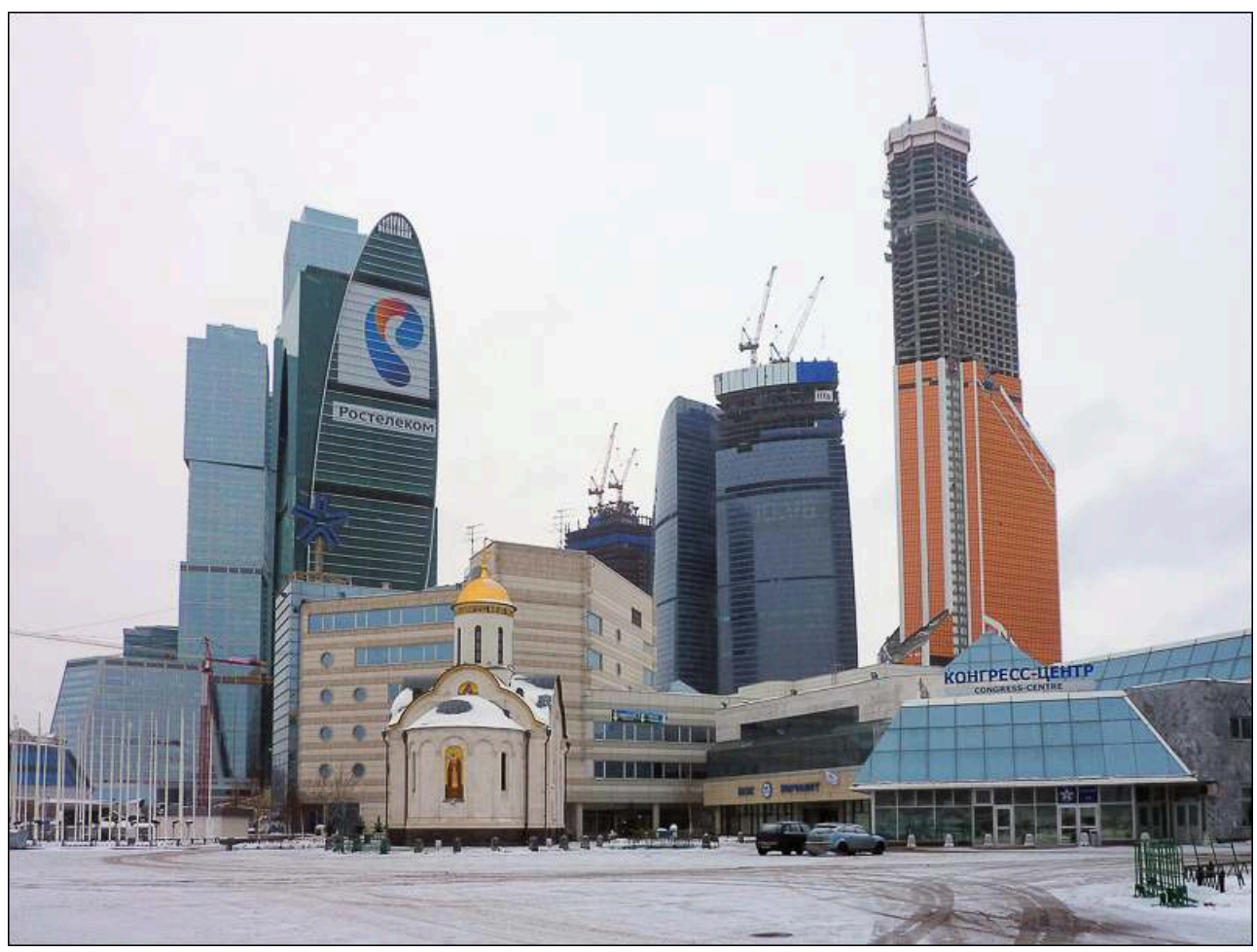

Auteur : E. Bonnet-Pineau, février 2012.

Surnommé le « Manhattan russe », ce futur centre d'affaires international donne lieu à un gigantesque chantier, le plus important d'Europe. Le projet fut initialement conçu dès 1992. Situé au bord de la Moskova à l'ouest du centre historique, il a connu plusieurs phases de ralentissement au gré des crises économiques et financières. 
Aujourd'hui, sur une surface de 100 hectares de terrains industriels déclassés, seuls 42 hectares seront construits. Le centre d'affaires doit accueillir «le complexe des villes capitales » constitué de deux tours identiques symbolisant Moscou et Saint-Pétersbourg (la tour de Moscou atteint $268 \mathrm{~m}$ de haut, celle de Saint-Pétersbourg $234 \mathrm{~m}$ ). La Douma municipale et tous les services du gouvernement de Moscou devraient y être regroupés. Le centre comprendra un ensemble de gratte-ciel offrant des bureaux aux entreprises privées, des hôtels et des centres commerciaux, des lieux de divertissement et des appartements. Quelques oligarques seraient les principaux actionnaires de cet ambitieux projet devant permettre à Moscou d'accéder au statut de ville mondiale auquel elle aspire.

Sur la photographie, la présence de l'église à la coupole redorée procède de la volonté d'ancrer à tout prix Moscou dans le passé. Il s'agit de l'église des Vieux croyants construite en 1921 grâce à des dons puis fermée en 1935. Elle a été restituée à l'Église orthodoxe en 1993 et ouverte au culte de nouveau en 1995. Tout en s'affichant comme la vitrine de la modernité, les autorités russes cherchent à réaffirmer les traditions nationales en s'appuyant sur l'Église orthodoxe, symbole du passé glorieux de l'Empire russe.

Illustration 7 - Le premier hypermarché Auchan : un centre commercial pour classes moyennes

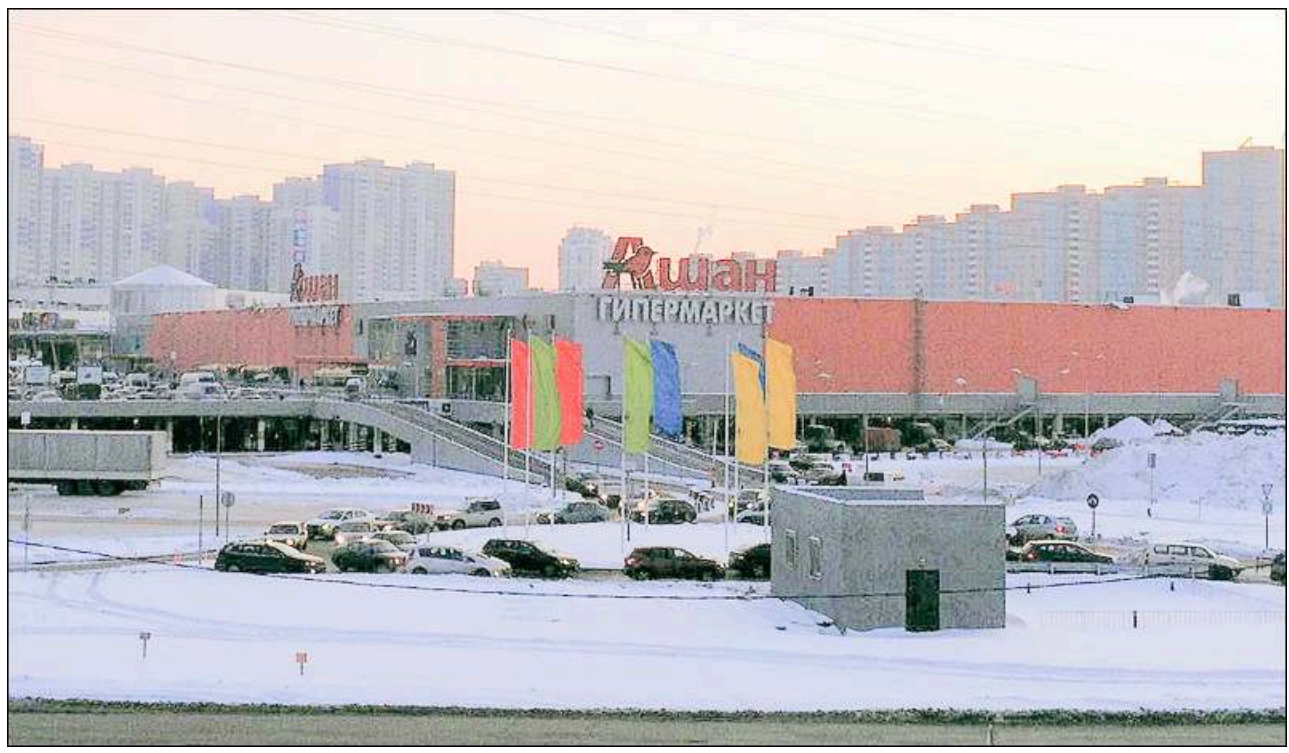

Auteur : E. Bonnet-Pineau, février 2012

La photographie montre le premier hypermarché ouvert en Russie en 2002, premier d'une série d'autres hypermarchés du même groupe français Auchan, situé près de l'aéroport de Cheremetievo. Il fait partie du centre commercial Mega, facilement accessible par la rocade MKAD. Ce complexe commercial, situé sur la commune de Khimki, est géré par le groupe Ikea qui s'y est implanté dès mars 2000 et il comporte d'autres grandes surfaces, des boutiques de sport et un complexe de cinéma. La Berd (Banque européenne pour la reconstruction et le développement) a participé au financement de ce centre commercial aux côtés d'investisseurs privés.

Moscou fut en effet pionnière en Russie pour le développement de telles grandes surfaces. Au cours des années 2000, aux grandes enseignes de la distribution internationale se sont ajoutés des groupes moscovites pour répondre aux attentes des 
classes moyennes. L'essor de tels centres commerciaux en périphérie n'a été rendu possible que par l'augmentation du parc automobile russe. En même temps, il a engendré à proximité une fièvre de construction de tours d'habitation.

Si le passage à l'économie de marché a favorisé dans un premier temps l'essor des classes moyennes, aujourd'hui, les «Nouveaux Russes» (appartenant aux classes moyennes supérieures ou aux ex-dignitaires du régime) travaillent beaucoup, voyagent, achètent des appartements dans des immeubles de grand standing ou vivent à l'écart du centre-ville dans des lotissements luxueux sécurisés installés le plus souvent près des nouveaux centres commerciaux Auchan.

Illustration 8 - Le G.U.M, un centre commercial pour les « Nouveaux Russes »

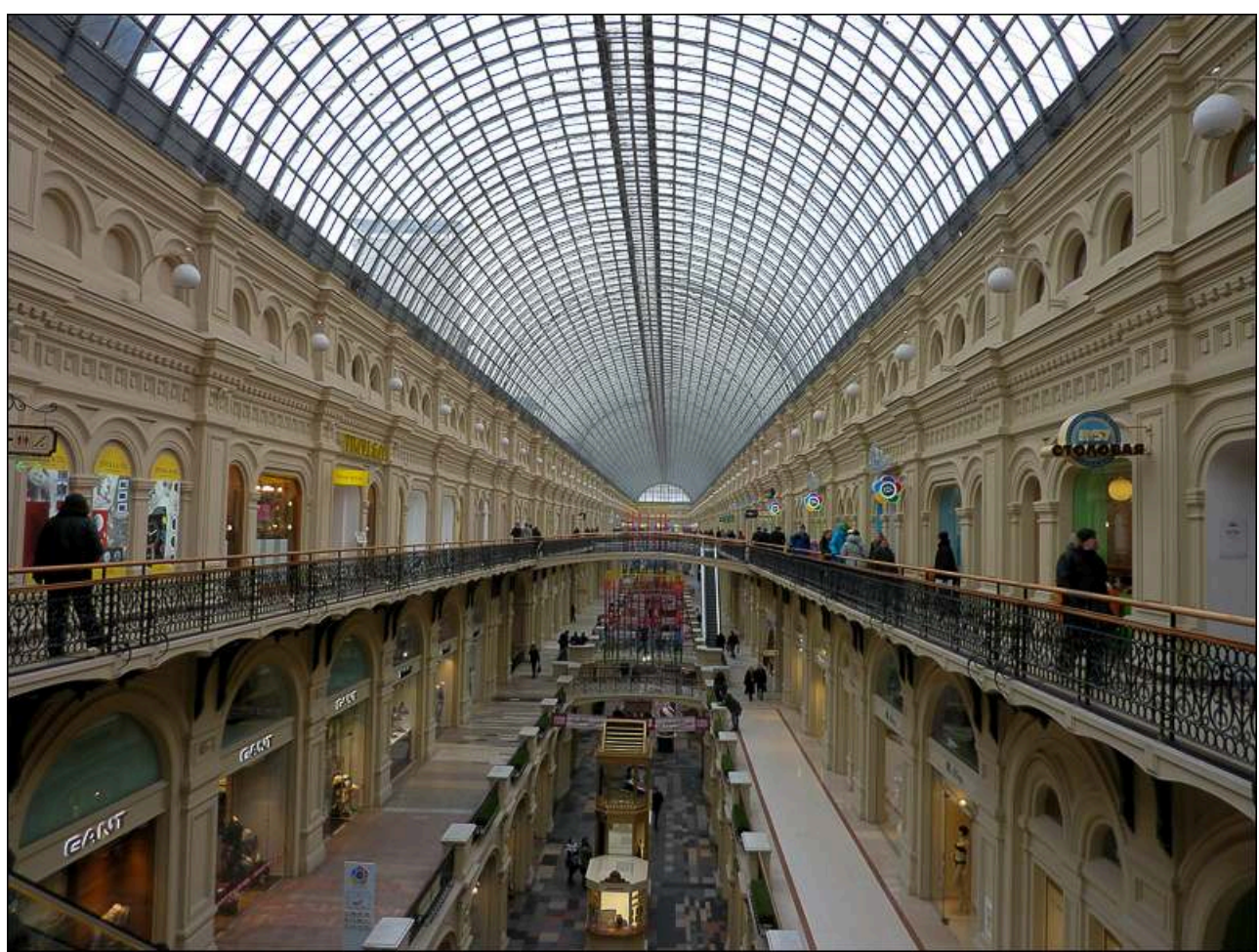

Auteur : E. Bonnet-Pineau, février 2012.

Situés dans le centre historique délimité par le Boulevard des jardins, plusieurs anciens grands magasins d'État comme le G.U.M. (ou Goum) et le Tsoum furent dès la décennie 1990 colonisés par le commerce de luxe.

Le Goum est maintenant le centre commercial le plus prestigieux ; il est situé au pied du Kremlin et l'une des façades débouche sur la Place Rouge. La structure métallique a été découpée en boutiques de luxe appartenant à de prestigieuses grandes marques occidentales. Il a été transformé en SARL au début des années 1990, à l'aube du renouveau du capitalisme. L'un des principaux actionnaires au début des années 2000 était le groupe Perekriostok, lié à l'ancien maire de Moscou, Iouri Loujkov. En 2004, ils ont vendu le paquet majoritaire qui, depuis, est contrôlé par le groupe italien Bosco di Ciliegi (marque de vêtements, fabricant officiel des maillots pour les équipes nationales de la Russie). Les magasins installés, sauf celui de Bosco, sont en location, mais les vrais propriétaires et actionnaires minoritaires ne sont pas bien connus. A l'image du capitalisme russe, les structures sont devenues très complexes. 

jeunesse de considérer ce centre comme un lieu de promenade.

\section{BIBLIOGRAPHIE}

Eckert D., 2007. Le monde russe. Hachette, coll. « Carré Géographie », 2e édition.

Fadeïeva M., 2010. Moscou, nouvelle "creative city". Courrier international, n¹025. http:// www.courrierinternational.com/article/2010/06/24/moscou-nouvelle-creative-city

Kolossov V., 2004. Comment Moscou se transforme en ville géante capitaliste. Revue internationale des sciences sociales, $n^{\circ} 181$.

Marchand P., 2010. Moscou. Autrement, Atlas coll. « Mégapoles ».

Nivat G., 2007. Les sites de la mémoire russe. Fayard.

\section{NOTES}

1. L'introduction, les photos 1 à 3 et 6 à 8 ainsi que leurs commentaires sont d'Elisabeth BonnetPineau. Les photos 4 et 5 ainsi que leurs commentaires sont d'Aurélien Delpirou.

\section{AUTEURS}

\section{ÉLISABETH BONNET-PINEAU}

Elisabeth Bonnet-Pineau, danieloster@wanadoo.fr, est géographe et consultante.

\section{AURÉLIEN DELPIROU}

Aurélien Delpirou, aurelien.delpirou@u-pec.fr, est maître de conférences à l'Institut d'Urbanisme de Paris (Université Paris-Est Créteil). 